Research Article

\title{
Diagnostic accuracy of fine needle aspiration cytology in nodular goitres with clinico-pathologic correlation
}

\author{
Prasad R. Gole, Bharat S. Kamath*, Kotagiri Madhava
}

\author{
Department of Surgery, Seth V. C. Gandhi and M. A. Vora Municipal General Hospital, Ghatkopar (E), Mumbai, \\ Maharashtra, India
}

Received: 11 July 2016

Accepted: 14 July 2016

\author{
*Correspondence: \\ Dr. Bharat S. Kamath, \\ E-mail: drbharatk@gmail.com
}

Copyright: ( ) the author(s), publisher and licensee Medip Academy. This is an open-access article distributed under the terms of the Creative Commons Attribution Non-Commercial License, which permits unrestricted non-commercial use, distribution, and reproduction in any medium, provided the original work is properly cited.

\begin{abstract}
Background: FNAC is now one of the initial diagnostic modalities for thyroid nodules and can be used in association with clinical diagnosis especially to rule out malignancy thereby avoiding unnecessary surgeries for benign pathologies. We evaluated the usefulness and diagnostic accuracy of fine needle aspiration cytology as an initial investigation in patients with nodular goitres by comparing it with histopathology.

Methods: A combined retrospective and prospective study comprising 61 patients admitted and operated for nodular goitres over a period of two years at a tertiary hospital was carried out. All patients were evaluated by detailed history, clinical examination, serum thyroid hormonal levels and FNAC which were divided into four categories namely benign, malignant, indeterminate and nondiagnostic. All the patients were subjected to surgery and the thyroid specimens were sent for histopathological evaluation. Finally clinical, cytological and histopathological correlation was analyzed and usefulness of FNAC in detecting malignancy was evaluated.

Results: In present study $47.54 \%$ cytologies were benign, 34.42 were indeterminate, $11.47 \%$ were malignant and $6.55 \%$ were nondiagnostic. For neoplastic lesions, FNAC had sensitivity of $84.37 \%$, specificity of $96 \%$, PPV of $96.42 \%$, NPV of $82.75 \%$ and diagnostic accuracy of $89.47 \%$. For carcinoma, FNAC had a sensitivity of $70 \%$, specificity of $100 \%$, PPV of $100 \%$, NPV of $94 \%$, false negative rate of $6 \%$, false positive rate of $0 \%$ and diagnostic accuracy of $94.73 \%$.

Conclusions: FNAC with its very high specificity, negative predictive value and diagnostic accuracy, approaching $100 \%$ can help to rule out malignancy in most cases of thyroid swellings and prevent unnecessary surgeries.
\end{abstract}

Keywords: Fine needle aspiration cytology, Solitary thyroid nodule, Nodular goiters, Thyroid malignancy

\section{INTRODUCTION}

Diseases of thyroid form a major share of head and neck surgeries. Thyroid swellings can be benign as well as malignant. Clinical diagnosis can be quite accurate. However it may not be adequate in some conditions such as multinodularity and malignancy. FNAC can be used in association with clinical diagnosis especially to rule out malignancy thereby avoiding unnecessary surgeries for benign pathologies. Also diseases of thyroid gland especially multinodular goiter due to iodine deficiency are prevalent in India., ${ }^{1,2}$ Also majority of these nodules are benign and don't require surgery and hence the main task is to distinguish between benign and malignant swellings with the help of clinical judgment and investigations. FNAC is now one of the initial diagnostic modalities in patients with thyroid nodules.

The aim was to study the epidemiologic factors, clinical presentation and assess the pattern of pathological outcomes in patients with nodular goiters. More importantly the focus was to evaluate the usefulness and 
diagnostic accuracy of fine needle aspiration cytology as an initial investigation in patients with nodular goitres by comparing it with histopathology.

\section{METHODS}

A combined retrospective and prospective study comprising 61 patients admitted and operated for nodular goitres over a period of 3 years at a tertiary hospital was carried out. All patients were evaluated in detail including age, sex, residence, history and duration of illness, hypo and hyperthyroid symptoms, pressure symptoms, any goitrogenic and radiation history and any other associated symptoms along with detailed clinical examination. A clinical diagnosis was formed on the basis of history and physical examination and thyroid status evaluated by serum TSH.

All patients underwent FNAC of their swelling and cytology results were divided into four categories namely benign, malignant, indeterminate and nondiagnostic. All the patients were subjected to surgery and the thyroid specimens were sent for histopathological evaluation. Finally clinical, cytological and histopathological correlation was analyzed and usefulness of FNAC in detecting malignancy was evaluated.

\section{RESULTS}

The highest age incidence $(32.78 \%)$ of nodular goitre was in the 31-40 year age group with a median age of 40 years. Also neoplastic lesions were most commonly seen in the age group of 41-50 years in males and 21-30 years in females. Overall the presentation of neoplastic lesion as per age group in both the sexes combined was maximum in the age group of 21 to 50 years.

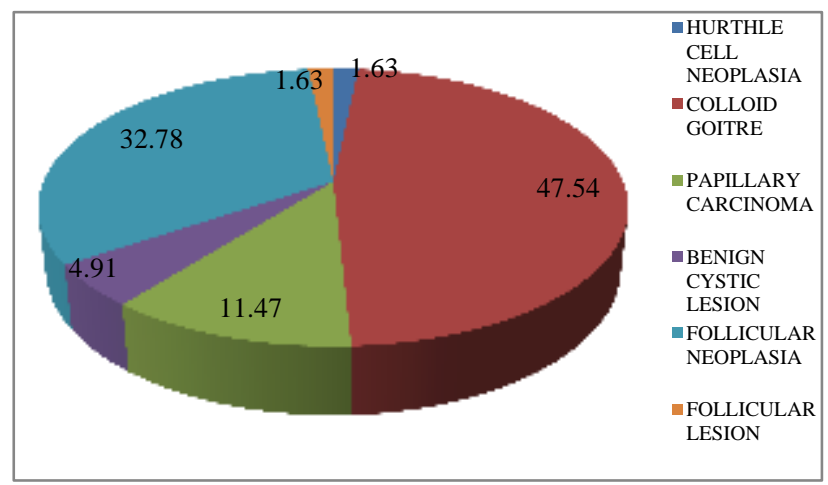

Figure 1: Percentage distribution of cytological diagnoses on FNAC in thyroid nodules.

The male to female ratio for thyroid swellings was $1: 2.58$, whereas for neoplastic lesions it was $1: 2.55$ and carcinomas was 1:9. Also the male to female ratio for adenoma, multinodular goiter and colloid goiter were $1: 1.75,1: 2.42$ and 1:4 respectively. The prevalence of neoplasia in males and females was almost the same in this study but that of carcinoma was around 5 times more in females.

The most presenting and common complaint in all patients was midline neck swelling, followed by dysphagia in 3 patients and dyspnoea in one patient with a massively enlarged gland and retrosternal extension. Also only 2 patients each had toxic symptoms and features of hypothyroidism respectively. Duration of symptoms in this study ranged from 15 days to 30 years, with $58.1 \%$ of patients having complaints less than a year in benign pathologies and $70 \%$ in malignant. Only 2 patients were hypothyroid on presentation and 2 had history of toxic symptoms but were euthyroid at the time of FNAC and surgery, while rest all patients were euthyroid clinically.

Table 1: Correlation of pre-operative FNAC and postoperative histopathology findings.

\begin{tabular}{|c|c|c|c|}
\hline \multirow{2}{*}{$\begin{array}{l}\text { FNAC } \\
\text { classification }\end{array}$} & \multirow{2}{*}{$\begin{array}{l}\text { Number } \\
\text { of } \\
\text { Patients }\end{array}$} & \multicolumn{2}{|c|}{ Histopathology } \\
\hline & & Benign & Malignant \\
\hline Benign & 29 & 28 & 1 \\
\hline Indeterminate & 21 & 19 & 2 \\
\hline Malignant & 7 & 0 & 7 \\
\hline Nondiagnostic & 4 & 4 & 0 \\
\hline
\end{tabular}

The clinical diagnosis correlated with the histopathological diagnosis in 46 cases. Fifteen out of 18 cases clinically diagnosed as multinodular goiters were found true, 29 out of 41 cases for solitary thyroid nodule and both the cases of colloid goiter were confirmed true on histopathology. Thus $75.40 \%$ of clinical diagnoses correlated with histopathological examination. After calculations, the accuracy of clinically diagnosing cases of multinodular goitres was found out to be $80.32 \%$ with sensitivity of $62.5 \%$ and specificity of $91.89 \%$.

Table 2: Efficacy of FNAC in diagnosis of carcinoma with correlation to histopathology.

\begin{tabular}{|llll|}
\hline \multirow{2}{*}{ FNAC } & \multicolumn{3}{c|}{$\begin{array}{l}\text { Histopathology } \\
\text { Carcinoma }\end{array}$} \\
\hline Carcinoma & 7 & $7(\mathrm{TP})$ & $0(\mathrm{FP})$ \\
\hline Benign+indeterminate & 50 & $3(\mathrm{FN})$ & $47(\mathrm{TN})$ \\
\hline Total & & 10 & 47 \\
\hline
\end{tabular}

Cytology was divided into four categories as per the Endocrine Society of India management guidelines for patients with thyroid nodules, as benign, malignant, indeterminate and nondiagnostic. In the study $47.54 \%$ cytologies were benign, 34.42 were indeterminate, $11.47 \%$ were malignant and $6.55 \%$ were nondiagnostic. The cytological diagnosis of follicular neoplasia and hurthle cell neoplasia were placed under indeterminate category. Two cystic lesions and one follicular lesion on FNAC were placed under nondiagnostic category. 
Findings of colloid on FNAC was considered benign. Most of the lesions were colloid goiter $(47.54 \%)$ or follicular neoplasia $(34.42 \%)$ on cytology. Papillary carcinoma was detected in $11.47 \%$ samples. For neoplastic lesions, FNAC had sensitivity of $84.37 \%$, specificity of $96 \%$, PPV of $96.42 \%$, NPV of $82.75 \%$ and diagnostic accuracy of $89.47 \%$. The prevalence of neoplasia as per detected by FNAC in present study was $56.14 \%$. However there were 4 FNACs which were nondiagnostic, and all were nonneoplastic lesions on histopathology. For carcinoma, FNAC had a sensitivity of $70 \%$, specificity of $100 \%$, PPV of $100 \%$, NPV of $94 \%$, false negative rate of $6 \%$, false positive rate of $0 \%$ and diagnostic accuracy of $94.73 \%$. The prevalence of malignancy as per detected by FNAC in present study was $17.54 \%$. Thus the sensitivity, specificity, positive predictive value, negative predictive value of FNAC in detecting malignancy in patients with clinically diagnosed solitary thyroid nodules was $77.77 \%, 100 \%$, $100 \%, 94.11 \%$ respectively and the diagnostic accuracy of FNAC in solitary thyroid nodule was $95.12 \%$ (Table 1). Out of the 29 FNACs labeled benign one turned out to be malignant $(3.44 \%)$. Prevalence of malignancy as per FNAC in the study was $12.28 \%$. There were no complications following FNAC in any of the patients.

All the patients were operated, of which 40 had hemithyroidectomy, 13 underwent subtotal thyroidectomy and 8 were subjected to total thyroidectomy. Of these, 4 patients developed hypothyroidism in the post-operative period and one had unilateral vocal cord palsy.

Multinodular goiter was the most commonly found pathology (39.34\%) followed by adenoma (36.06\%). Among the malignant pathologies $90 \%$ of the carcinomas were papillary and $10 \%$ were follicular. One papillary carcinoma was incidentally detected in a lesion of multinodular goitre. Except for one hurthle cell adenoma all other adenomas were follicular type. The prevalence of neoplasia in the present study group as per histopathology is $52.45 \%$ and that of carcinoma is $16.39 \%$.

\section{DISCUSSION}

In the series, maximum patients $(32.78 \%)$ were in the 31 40 age groups and $68.84 \%$ in the $21-50$ years age group with a median age of 40 years, which corroborates with world literature and as reported by Sengupta et al. ${ }^{3}$ As per literature, the 20-40 years group is more susceptible for hormonal changes leading to increase in the levels of thyroid binding globulins and increase requirements for iodine. So this might be responsible for prevalence of thyroid disorders more in this age group. Neoplastic cases were most commonly found in the age group of 21-30 years, whereas carcinoma predominated in the 31-40 years age group. In their study, Likhar et al reported that the most common age group for malignant thyroid lesions was $30-60$ years. ${ }^{4}$ Overall females were predominantly affected, with a male to female ratio of 1: 2.75 whilst for malignancy it was 1:9 which is comparable with other studies. $^{5,6}$

All the patients $(100 \%)$ had swelling in the front of the neck as their primary complaint and pressure symptoms as the next most common symptom. Most patients presented within a year of the duration of their disease. In their series, Sengupta et al reported that $93.2 \%$ patients presented with midline neck swelling and pain as the next most common symptom. The duration of complaints varied from 6 months to 3 years in majority of the patients. ${ }^{3}$ Present series, had $93.44 \%$ of the patients detected as euthyroid on serum thyroid profile which is comparable with the $90 \%$ of patients in the study by Sengupta et al. ${ }^{3}$

The most common clinical diagnosis was of solitary thyroid nodules $(67.21 \%)$. However, about a third of these turned out to be multinodular goitres which could have been detected with ultrasound imaging increasing the diagnostic accuracy in detecting multiple nodules and thereby a benign condition. Out of the 18 clinically diagnosed cases of multinodular goitres, 15 were proved true, demonstrating that clinical diagnosis has sensitivity of $62.5 \%$, specificity of $91.89 \%$ and diagnostic accuracy of $80.32 \%$ in detecting multinodularity. However $5.55 \%$ of these clinically diagnosed multinodular goites turned out to be malignant and hence these cases would require proper monitoring and follow-up. Also in these cases, doppler ultrasound would help in detecting these small number of malignant lesions by showing increased vascularity in a nodule. Gerry $\mathrm{H}$ et al compared palpation and ultrasonography (USG) for solitary nodule and concluded that around $50 \%$ of solitary nodules are found to be multinodular on USG, thus concluding that clinical palpation is less sensitive than USG and reliable only if the nodule is $>1 \mathrm{~cm} .^{7}$ In nodular thyroid disease, USG can accurately determine the number and size of the nodules. It is also extremely useful in guiding in FNAC and USG guided FNAC was recommended as a routine for the evaluation of thyroid nodule by Nilakanth A et al. ${ }^{2}$

Also in the series, $5.5 \%$ of the clinically diagnosed cases of multinodular goitres turned out to be malignant while $21.95 \%$ cases of solitary nodule turned out to be malignant thus proving that malignancy is far more common in solitary nodules. On review of some studies, it is concluded that frequency of carcinoma in multinodular goitres is half of that found in solitary nodule but risk of malignancy is the same in both and hence even multinodular cases should be evaluated and regularly followed up. 8,9

In the series, FNAC was done for all the patients and its diagnostic accuracy (94.73\%), sensitivity, specificity, positive predictive value and negative predictive value corroborated with other studies worldwide. ${ }^{3,8,10}$ Gharib $\mathrm{H}$ had concluded that the accuracy of FNAC analysis in solitary nodules approaches $95 \%$ in the differentiation of 
benign from the malignant which corroborates with the diagnostic accuracy $(95.12 \%)$ in the series. ${ }^{11}$ The false negative and positive rate of FNAC in the series was $6 \%$ and $0 \%$ respectively which is consistent with world literature. ${ }^{5,6}$ False negative result may occur because of sampling error or misinterpretation of cytology and are of great concern as they indicate the potential to miss a malignant lesion. ${ }^{12}$ However, in this study false negative rate was only $6 \%$.

FNAC was also evaluated in its accuracy to detect neoplasia. The sensitivity, specificity, PPV, NPV and diagnostic accuracy of FNAC in the present study corroborates with other series reported by Gupta $\mathrm{M}$ et al and Mandrekar S et al. ${ }^{10,13}$ Prevalence of malignancy on FNAC in the present study group was $12.28 \%$ and on HPE was $16.39 \%$. In their series, Kumar $S$ et al and Sengupta A et al found the prevalence of malignancy on FNAC and HPE to be $7.86 \%, 10.11 \%, 9.55 \%$ and $11.24 \%$ respectively. ${ }^{3,14}$ The prevalence of papillary carcinoma in the study was $90 \%$ and follicular carcinoma was $10 \%$. This is in comparable with that given in literature. $^{15,16}$

Imaging was not evaluated in our study. Ultrasound of the neck is very useful tool for evaluating thyroid swellings especially solitary nodules, and when FNAC findings are nondiagnostic all cases of suspected or confirmed thyroid nodules should be evaluated by ultrasound. Watters et al concluded in their study that FNAC and USG are complimentary. They said USG was superior to FNAC in evaluating nodular goitre as it can accurately determine the number and size of the nodules. ${ }^{17}$ It is also extremely useful in visual guidance of FNAC and USG guided FNAC was recommended as a routine for the evaluation of thyroid nodule by Nilakanth A et al. ${ }^{2}$ However despite widespread use USG guided FNAC, this modality is also associated with a significant rate of initial nondiagnostic cytology approaching 20$25 \% .{ }^{18}$ Meko et al found high false negative rates in the large $(>3 \mathrm{~cm})$ nodules for malignancy and because of the high prevalence of the malignancy in large nodules, they concluded to strongly consider lobectomy in these cases even if FNAC is showing benign lesion. ${ }^{19}$

In the series, FNAC was done without USG guidance and it was not repeated in the nondiagnostic cases which actually would have had further increased accuracy of the diagnosis, turning out to be a drawback. All patients in the study underwent surgery, thereby ensuring histopathological specimens for comparison with cytology. This provided for an adequate correlation between clinical, cytological and pathological aspects of the disease.

\section{CONCLUSION}

Nodular thyroid goitres are more common in females and in the age group of 31-40 years and are predominantly euthyroid. Also most malignant lesions too occurs in the same age group and would present in less than a year after the swelling first appeared. Almost a third of clinically diagnosed solitary thyroid nodule proves to be multinodular on ultrasound, proving it to be useful for confirmation. Though the chances of malignancy in multinodular goitre are low, even patients on conservative treatment should be monitored closely as malignancy still cannot be ruled out in them and doppler ultrasound may be of help for detecting malignancy in these as well as for monitoring. FNAC with its very high specificity, negative predictive value and diagnostic accuracy, approaching $100 \%$ can help to rule out malignancy in most cases of thyroid swellings and prevent unnecessary surgeries. However FNAC has a false negative rate of $2-7 \%$ which can lead to malignancy being missed in some cases. Hence the only confirmatory test to rule out malignancy is excision and histopathological evaluation. Papillary carcinoma seems to be the most common thyroid malignancy.

\section{Funding: No funding sources \\ Conflict of interest: None declared}

Ethical approval: The study was approved by the institutional ethics committee

\section{REFERENCES}

1. Afroze N, Kayam N, Hassan SH. Role of FNAC in the diagnosis of palpable thyroid lesion. Indian Journal of Pathology and Microbiology. 2002;45(3):241-6.

2. Nilkantha A, Venkatesh, Raghavan D, Datta R, Sharma V. USG- its role in nodular thyroid disease. Indian Journal of Otolaryngology and Head and Neck Surgery. 2007;59(4):332-5.

3. Sengupta A, Pal R, Kar S, Zaman FA, Basu M, Pal S. Clinicopathological correlates of incidentally revealed thyroid swellings in Bihar. Indian Journal of Pharmacy and Bioallied Sciences. 2012;4(1):515 .

4. Likhar KS, Hazari RA, Gupta SG, Shukla CL. Diagnostic accuracy of FNAC in thyroid lesion: a hospital based study. Thyroid Res Pract. 2013;10:68-71.

5. Layfield LJ, Rechman A, Bottles K, Giulano A. Clinical determinants for the management of thyroid nodules by FNAC. Arch Otolaryngol Head and Neck Surgery. 1992;182:717-21.

6. Liel Y, Arid S, Barchana M. Long term follow up of patients with initially benign thyroid FNAC. Thyroid. 2001;11:775-8.

7. Gerry HT, Hossein G, Carl C. Comparison between USG and palpation for solitary nodules. Archives of Internal Medicine. 1995;155(2):2418-23.

8. Nepali R, Banita V, Thakur G. Comparative study of FNAC and histopathology in the diagnosis of thyroid swelling. The Internet Journal of Head and Neck Surgery. 2012;5(2):1-6. 
9. Bagga PK, Mahajan NC. FNAC of thyroid swelling: how useful and accurate is it? Indian Journal of Cancer. 2010:47(4);437-42.

10. Gupta M, Gupta S, Gupta VB. Correlation of FNAC with histopathology in diagnosis of solitary thyroid nodule. Journal of Thyroid Research. 2010;1:1-5.

11. Gharib H. Fine needle aspiration biopsy of thyroid nodules: advantage, limitations and effects. Mayo Clinic Proc.1994;69(1):44-9.

12. Hall TL, Layfield LJ, Phillipe A, Rosenthal DL. Source of diagnostic error in FNAC of thyroid. Cancer. 1989;63:718-25.

13. Mandrekar SR, Nadkarni NS, Pinto RG, Menzes S. Role of FNAC as the initial mode of investigation in a thyroid lesion. Acta Cytological. 1995; 39(5):898904.

14. Kumar S, Aquil S, Abdullah D. Role of FNAC in thyroid disease. Journal of Surgery Pakistan. 2008;13(1):22-5.

15. Krukowski ZH. The thyroid and parathyroid glands. In: Norman S Williams, Christopher J K Bulstorde,
P Ronan O Connel- Editors. Bailey and Love's Short Practice of Surgery. $25^{\text {th }}$ Edition. Hodder Arnold Publishers;2008:771-806.

16. Gregory PS, Orlo HC, Jon A, Van H. Malignancy of the thyroid. Schwartz Principles of Surgery.7th edition. Mc Graw Hill publishers;1999:1661-1711.

17. Watters DA, Ahuja AT, Chik EW, King WK. Role of USG in the management of thyroid nodule. American Journal of Surgery. 1992;164(6):654-7.

18. Tabaqchali MA, Hansen JM, Jhonson SJ, Wadhera V, Lennard TWJ, Proud G. Thyroid aspiration cytology in Newcastle: a six year cytology/histology correlation study. Ann of Royal Coll Surg Engl. 2000;82:149-55.

19. Meko JB, Norton JA. Large cystic/solid nodules a potential false negative fine needle aspiration. Surgery. 1995;118(6):996-1003.

Cite this article as: Gole PR, Kamath BS, Madhava K. Diagnostic accuracy of fine needle aspiration cytology in nodular goitres with clinico-pathologic correlation. Int Surg J 2016;3:1077-81. 